\title{
ALTERATION OF VOLATILE CHEMICAL COMPOSITION IN TOBACCO PLANTS DUE TO GREEN PEACH APHID (MYZUS PERSICAE SULZER) (HEMIPTERA: APHIDIDAE) FEEDING
}

\author{
SONG, Y.Z. ${ }^{1,2,3}-$ GUO, Y. Q. ${ }^{1,2}-$ CAI, P. M. ${ }^{2,3}-$ CHEN, W. B. ${ }^{1,2}$ - LIU, C. M. ${ }^{1,2^{*}}$ \\ ${ }^{I}$ Biological Control Research Institute, College of Plant Protection, Fujian Agriculture and \\ Forestry University, Fuzhou 350002, China \\ e-mail:1023554932@qq.com (Song, Y.Z.); 1220576278@qq.com (Guo, Y.Q.); \\ 447908983@qq.com (Chen, W. B.) \\ ${ }^{2}$ State Key Laboratory of Ecological Pest Control for Fujian and Taiwan Crops, Fuzhou \\ 350002, China \\ e-mail: caipumo@qq.com (Cai, P.M.) \\ ${ }^{3}$ Department of Horticulture, College of Tea and Food Science, Wuyi University, Wuyishan \\ 354300, China \\ *Corresponding author \\ e-mail: cmliu@fjau.edu.cn; phone: +86-0591-8378-9420; fax: +86-0591-8378-9421 \\ (Received $10^{\text {th }}$ Jul 2020; accepted $6^{\text {th }}$ Oct 2020)
}

\begin{abstract}
In response to insect pest herbivory, plants can generate volatile components that may serve multiple roles as communication signals and defence agents in a multitrophic context. In the present study, the volatile profiles of tobacco plants Nicotiana tabacum L., with and without infestation by sap-sucking aphids Myzus persicae Sulzer, were measured by gas chromatography-mass spectrometry (GC-MS). The results revealed that a total of 10 compounds were identified from healthy tobacco plants, and a total of 14 and 16 compounds were isolated from aphid-infested tobacco plants at 24 and 48 hours after infestation, respectively. Compared to intact tobacco plants, tridecane, 1h-3a,7-methanoazulene, tetradecane, pentadecane, hentriacontane, nonane, 1,8-nonadien-3-ol, heneicosane, sulfurous acid, limonene, cedrene and dichloro acetaldehyde were newly produced in aphid-infested tobacco plants, followed by five special components that only emitted from tobacco plants at 48 hours after aphidinfestation, and were similar to aliphatic compounds. However, the abundance of aromatic compounds in infested tobacco plants was significantly reduced compared to intact plants. The science of HIPVs belongs to chemical ecology, which possess a powerful potential for exploiting effective and practical infochemical-based methods to regulate the population of natural enemies, and enhance the resistance of crops in an agricultural production system.
\end{abstract}

Keywords: plant-insect interactions, aphid, Nicotiana tabacum, indirect defense, GC-MS

\section{Introduction}

Plants are constantly threatened by diverse abiotic and biotic stresses, in which biotic stress caused by herbivore damage is an important restriction in the yield and quality of crops of economic importance (Erb and Reymond, 2019). Nevertheless, plants have evolved direct and indirect defence mechanisms in response to herbivory via a series of morphological, biochemical and molecular alterations (Sharma et al., 2009; War et al., 2011). Direct defences against herbivorous insects could be physical or chemical, and contain morphological obstruction such as cell wall lignification, trichomes and silica deposition, and the production of toxic chemicals, including phenolics, alkaloids and terpenoids, which play as deterrents, repellents, anti-nutrients and digestion inhibitors (Kessler et al., 2001; Sharma et al., 2009; War et al., 2011; Aljbory and Chen, 2018). 
Indirect defences, which are modulated by the emission of volatile components derived from attacked plants, are known as herbivore-induced plant volatiles (HIPVs) (Kessler et al., 2001; Arimura et al., 2009; Aljbory and Chen, 2018), and refers to the recruitment of natural enemies antagonistic to phytophagous insects. Furthermore, this also enhances the host-seeking ability of carnivores, thereby contributing to the better suppression of pests (Kessler et al., 2001; Cai et al., 2020).

Understanding the chemical ecology of the tritrophic interactions of natural enemies, pests and host plants plays an important role in the exploitation of effective and practical integrated pest management tactics, in which the distribution and abundance of natural enemies could be operated by infochemicals (Hilker and Fatouros, 2015). Herbivore-induced plant volatiles are the 'words' of a complex language that are not only used to communicate between attacked plants and natural enemies of infesting herbivores, but also informs neighbouring healthy plants of an impending attack, in addition to communicating between different parts of the same plant via intraplant signalling (Heil, 2008; Arimura et al., 2009; Karban, 2011; Aljbory and Chen, 2018; Erb and Reymond, 2019). HIPVs are released not only from the damaged parts of the plant, but also from intact parts, which amplifies the detectability of the chemical signal (Dicke et al., 2009). To date, approximate 2,000 volatile components emitted in response to herbivory have been identified from 900 plant families (War et al., 2011). A certain amount of volatile components is released by plants into the atmosphere from aboveground parts or into the soil from the roots. However, once damaged, the blend of these emitted volatiles would be altered (Kessler et al., 2001; Dicke et al., 2009; Aljbory and Chen, 2018). The plant protection implications for applying HIPVs, as an assistance within the framework of conservation biological control (CBC), are of significant importance (Turlings and Ton, 2006; Cai et al., 2020).

Myzus persicae Sulzer (Hemiptera: Aphididae), which is known as green peach aphid, is a cosmopolitan and highly polyphagous aphid species with high agronomical and ecological importance. This is mainly due to its ability of infesting plants distributed at 40 different families, which vector over 100 plant viral pathogens, and results in considerable quality and yield losses of crops (Martin et al., 1997; Blackman and Eastop, 2017; Hong et al., 2019). Myzus persicae has evolved its resistance to at least 70 synthetic components, and developed at least seven independent resistance mechanisms to several classes of insecticides, making it extremely difficult to suppress the population of this pest (Silva et al., 2012; Bass et al., 2014). To date, alternative strategies that can effectively control this aphid pest have been insufficient and highly sought after. Furthermore, numerous research groups have focused their attention on the substances of natural origins as pest control agents, which mainly concentrate on plant secondary metabolites that are considered as elementary compounds of natural plant resistance against insect pests (Wróblewska-Kurdyk et al., 2015; Philippi et al., 2015; Phuong et al., 2015; Jackowski et al., 2017).

Herbivory by phytophagous pests can elicit the indirect defence responses of plants, in which volatile components are released. These warn neighbouring plants and recruit natural enemies, such as parasitoids and predators (Hilker and Meiners, 2006; Aljbory and Chen, 2018). However, suppression can also occur (Peñaflor et al., 2011). Plants that can generate HIPVs in response to herbivory possess the metric of protecting themselves early, and before colonized pests can further attack the plant. As a plant with a short life cycle, tobacco Nicotiana tabacum L. (Tubiflorae: Solanaceae) would benefit from the indirect defence responses of plants induced by aphid infestation. However, the 
qualitative and quantitative variation in the content of allelochemicals emitted from infested tobacco plants remain poorly known. Thus, the present study aims to determine the response of tobacco plants to herbivory by sap-sucking aphids, and specifically determine whether $M$. persicae feeding would induce tobacco plants to alter their headspace volatiles profile.

\section{Materials and Methods}

\section{Aphids rearing}

The research was conducted from August to November 2019 at the Institute of Biological Control, Fujian Agriculture and Forestry University (IBC, FAFU), Fuzhou, Fujian, China. The initial colony of $M$. persicae was collected from vegetable fields located at IBC, FAFU. Rearing was maintained on tobacco plants (cultivars K326), within $50-\mathrm{cm}^{3}$ mesh cage conditions of $25 \pm 1^{\circ} \mathrm{C}, 75 \pm 5 \%$ relative humidity (RH), and a L:D photoperiod of 14:10 hours. Emerging $M$. persicae adults were transferred to new cages with non-infested tobacco plants at the 4-6 leaf stage every two weeks.

\section{Tobacco cultures}

The tobacco (cultivars K326) seeds were supplied by Nanping Tobacco Co. Ltd., Fujian, China, and planted following the protocols described by Wei et al. (2003). The tobacco plants were grown from seed in plastic trays (length $\times$ width $\times$ height: $30 \times 25 \times 10 \mathrm{~cm})$ in a growth chamber with a natural lighting under the conditions of $25 \pm 1{ }^{\circ} \mathrm{C}$ and $70 \pm 5 \%$ relative humidity $(\mathrm{RH})$. The seedlings were individually transferred into a plastic bowl (upper diameter: $9 \mathrm{~cm}$, bottom diameter: $6.5 \mathrm{~cm}$, height: $7 \mathrm{~cm}$ ) at the two-leaf stage, and deposited in $50-\mathrm{cm}^{3}$ insect-proof mesh cages. The tobacco plants were supplied with water, as needed, fertilized every two weeks at a dose of $0.05 \mathrm{~g}$ $(\mathrm{N}: \mathrm{P}: \mathrm{K}=20: 20: 20)$ per plant, and applied for the assays at the stage of four fully grown leaves.

\section{Preparation of aphid-infested tobaccos}

At the four-leaf stage, the tobacco plants were infested with 400 third- or fourthinstar aphids at a density of 100 aphids/leaf, and were thereafter encased in individual insect-proof mesh cages after inoculation. Then, the aphids were removed from the tobacco plants at two hours after infestation, and the infested tobaccos were used for volatile collection at 24 and 48 hours after aphid-infestation, respectively. The control plants were similarly enclosed, but no aphids were introduced and maintained until these were used for volatile collection. Three plants were used for each treatment.

\section{Volatiles collection}

The collection of feeding-induced volatiles by $M$. persicae on tobacco plants was conducted using the headspace absorption method in a static environment. The whole tobacco plant was deposited in a $30 \times 50 \mathrm{~cm}^{2}$ polyacetate bag for volatiles collection (Anfora et al., 2009). The charcoal-filtered air from the headspace of the bag was pushed at a rate of $200 \mathrm{~mL} / \mathrm{min}$ through a sorbent column $(50 \mathrm{mg}$ Porapak Type Q, Sigma-Aldrich) connected to an air pump through Teflon tubing. The volatile collections for each treatment were replicated for three times, according to the same protocol. The collected volatiles were eluted from the sorbent column using $500 \mu \mathrm{L}$ of 
hexane (purity $>99 \%$, Sigma-Aldrich) as solvent desorption at room temperature. The collections were concentrated to $50 \mu \mathrm{L}$ by slow nitrogen stream, and $0.5 \mu \mathrm{g}$ of nonyl acetate (purity $\geq 99 \%$, Sigma-Aldrich) was added as the internal standard. The extracts were preserved in $2-\mathrm{mL}$ brown vials at $-20^{\circ} \mathrm{C}$ until utilized for the GC-MS analysis.

\section{Chemical analysis}

The specific headspace volatiles that blended within the tobacco plants were identified by gas chromatography-mass spectrometry, and analyzed in a 7890A GC equipped with a 5975C mass selective detector. The splitless mode comprised of the DB-5MS column (Agilent Technologies Inc., Folsom, CA, $60 \mathrm{~m} \times 0.25 \mathrm{~mm}$ inner diameter, $0.25-\mathrm{mm}$ film thickness) programmed to increase from $50^{\circ} \mathrm{C}$, which was held for two minutes, up to $80^{\circ} \mathrm{C}$ at $1{ }^{\circ} \mathrm{C} / \mathrm{min}$ for one minute, up to $90^{\circ} \mathrm{C}$ at $2^{\circ} \mathrm{C} / \mathrm{min}$, up to $110^{\circ} \mathrm{C}$ at $5^{\circ} \mathrm{C} / \mathrm{min}$, up to $130^{\circ} \mathrm{C}$ at $2^{\circ} \mathrm{C} / \mathrm{min}$, and finally, up to $200^{\circ} \mathrm{C}$ at $5^{\circ} \mathrm{C} / \mathrm{min}$ for two minutes, with helium (purity $>99.99 \%$ ) as the carrier gas at a flow rate of $1 \mathrm{~mL} / \mathrm{min}$. A $70-\mathrm{eV}$ of electron impact energy was utilized for sample ionization. The putative identification of the volatile compounds was characterized by comparing with synthetic standards, taking into consideration the GC retention parameters, and comparing with the mass spectra through the Wiley 7N (John Wiley, NY) and NIST 11 (Gaithersburg, MD) mass spectral libraries. The quantification of the most abundant components in each extract was conducted by comparing the peak areas with that of the internal standard, according to the protocols mentioned by Faccoli et al. (2011).

\section{Data analysis}

The data analysis was conducted using SPSS v.17.0 (SPSS, Inc., Chicago, IL, USA). The effects of the treatment on the percentage of each identified volatile compound was analyzed using Tukey's honestly significant differences (HSD) test for multiple mean comparisons after one-way ANOVA.

\section{Results}

As shown in Table 1, 10 compounds were identified from healthy tobacco plants and 19 compounds were identified from tobacco plants that suffered from the infestation by $M$. persicae. A total of 14 and 16 compounds were isolated from the aphid-infested tobacco plants at 24 and 48 hours after herbivory, respectively. For 24 and 48 hours, benzene, nonadecane and tetracosane both vanished, when compared to the intact tobacco plants. Compared to the intact tobacco plants, tridecane, 1h-3a,7-methanoazulene, tetradecane, pentadecane, hentriacontane, nonane and 1,8-nonadien-3-ol were newly produced in aphid-infested tobacco plants with 24 hours after infestation. Otherwise, tridecane, 1h-3a,7-methanoazulene, tetradecane, 1,8-nonadien-3-ol, heneicosane, sulfurous acid, limonene, cedrene and dichloro acetaldehyde newly emerged in aphid-infested tobacco plants within 48 hours after infestation. Among these newly generated volatile components, pentadecane, hentriacontane and nonane were only isolated from tobacco plants with aphid infestation for 24 hours. Furthermore, heneicosane, sulfurous acid, limonene, cedrene and dichloro acetaldehyde were the special components only emitted from tobacco plants with the 48-hour aphid-infestation. Among all the identified compounds, the abundance of undecane and 1,8-nonadien-3-ol ascended as the aphid infestation time 
increased, while the abundance of 1h-3a,7-methanoazulene peaked at tobacco plants within 24-hours of aphid-infestation, which was significantly higher than that of healthy tobacco plants. No significant differences in the content of other identified compounds were detected in the headspace composition profile between healthy plants and infested plants.

Table 1. Mean ( \pm SE) percentage of main volatile components of tobacco plants after infection by $M$. persicae for various durations

\begin{tabular}{|c|c|c|c|c|c|}
\hline \multirow{2}{*}{ ID } & \multirow{2}{*}{ Compounds } & \multicolumn{3}{|c|}{ Hours after infestation } & \multirow{2}{*}{$F$ and $P$} \\
\hline & & $\mathbf{0}$ & 24 & 48 & \\
\hline 1 & $(+)$ - $\alpha$-Funebrene & $4.68 \pm 1.40$ & $8.14 \pm 1.86$ & $5.64 \pm 1.22 \mathrm{NS}$ & $\mathrm{F} 2,6=1.38, \mathrm{P}=0.32$ \\
\hline 2 & Methylene chloride & $3.65 \pm 0.70$ & $4.25 \pm 0.79$ & $3.55 \pm 0.49 \mathrm{NS}$ & $\mathrm{F} 2,6=0.32, \mathrm{P}=0.74$ \\
\hline 3 & trans-Decalin & $1.54 \pm 1.54$ & $2.80 \pm 1.40$ & $2.08 \pm 2.08 \mathrm{NS}$ & $\mathrm{F} 2,6=0.14, \mathrm{P}=0.87$ \\
\hline 4 & Naphthalene & $5.17 \pm 2.74$ & $2.62 \pm 0.74$ & $2.88 \pm 1.72 \mathrm{NS}$ & $\mathrm{F} 2,6=0.54, \mathrm{P}=0.61$ \\
\hline 5 & Undecane & $5.23 \pm 0.18 b$ & $5.69 \pm 1.04 \mathrm{~b}$ & $10.54 \pm 0.95 \mathrm{a}$ & $\mathrm{F} 2,6=12.96, \mathrm{P}=0.04$ \\
\hline 6 & Dodecane & $2.73 \pm 1.16$ & $5.23 \pm 1.38$ & $5.83 \pm 1.15 \mathrm{NS}$ & $\mathrm{F} 2,6=1.78, \mathrm{P}=0.25$ \\
\hline 7 & Octane & $1.63 \pm 0.82$ & $2.21 \pm 0.16$ & $1.49 \pm 0.81 \mathrm{NS}$ & $\mathrm{F} 2,6=0.33, \mathrm{P}=0.73$ \\
\hline 8 & Benzene & $4.47 \pm 4.47^{\mathrm{NS}}$ & - & - & $F_{2,6}=1.00, P=0.42$ \\
\hline 9 & Nonadecane & $0.35 \pm 0.35^{\mathrm{NS}}$ & - & - & $F_{2,6}=1.00, P=0.42$ \\
\hline 10 & Tetracosane & $0.51 \pm 0.52^{\mathrm{NS}}$ & - & - & $F_{2,6}=1.00, P=0.42$ \\
\hline 11 & Tridecane & - & $2.55 \pm 0.76$ & $3.96 \pm 1.74^{\mathrm{NS}}$ & $F_{2,6}=3.35, P=0.11$ \\
\hline 12 & 1H-3a,7-Methanoazulene & - & $3.15 \pm 0.76$ & $1.37 \pm 0.84^{\mathrm{NS}}$ & $F_{2,6}=5.79, P=0.40$ \\
\hline 13 & Tetradecane & - & $1.24 \pm 0.86$ & $1.42 \pm 0.38^{\mathrm{NS}}$ & $F_{2,6}=2.05, P=0.21$ \\
\hline 14 & 1,8-Nonadien-3-ol & - & $1.13 \pm 0.32 b$ & $3.93 \pm 0.26 \mathrm{a}$ & $F_{2,6}=71.43, P=0.00$ \\
\hline 15 & Pentadecane & - & $0.50 \pm 0.50^{\mathrm{NS}}$ & - & $F_{2,6}=1.00, P=0.42$ \\
\hline 16 & Hentriacontane & - & $1.61 \pm 0.80^{\mathrm{NS}}$ & - & $F_{2,6}=4.00, P=0.08$ \\
\hline 17 & Nonane & - & $1.31 \pm 0.93^{\mathrm{NS}}$ & - & $F_{2,6}=2.00, P=0.22$ \\
\hline 18 & Heneicosane & - & - & $0.52 \pm 0.29^{\mathrm{NS}}$ & $F_{2,6}=3.08, P=0.12$ \\
\hline 19 & Sulfurous acid & - & - & $0.71 \pm 0.41^{\mathrm{NS}}$ & $F_{2,6}=2.96, P=0.13$ \\
\hline 20 & Limonene & - & - & $1.20 \pm 1.20^{\mathrm{NS}}$ & $F_{2,6}=1.00, P=0.42$ \\
\hline 21 & Cedrene & - & - & $1.08 \pm 1.08^{\mathrm{NS}}$ & $F_{2,6}=1.00, P=0.42$ \\
\hline 22 & Dichloro acetaldehyde & - & - & $0.37 \pm 0.37^{\mathrm{NS}}$ & $F_{2,6}=1.00, P=0.42$ \\
\hline
\end{tabular}

Note: "-" refers to non-detectable. NS indicates that there is no significant difference between the three treatments. The different letters indicate the significant differences (Tukey's HSD test after ANOVA, $\mathrm{P}<0.05$ )

As exhibited in Figures 1 and 2, the contents of emitted volatiles from tobacco plants were elevated as the aphid-infestation time increased, and the content of isolated volatile compounds from the 48-hour aphid infestation was significantly higher than that in intact tobacco plants $\left(F_{2,6}=6.32, P=0.03\right)$ (Fig. 1). Similarly, the abundance of aliphatic compounds also exhibited the same upward trend, and a significant difference was also observed among tobacco plants with different aphid-infestation durations $\left(F_{2,6}=11.58, P=0.01\right)$. Furthermore, the content of aromatic compounds emitted from infested tobacco plants significantly decreased, when compared to intact plants $\left(F_{2,6}=10.97, P=0.03\right)$. This was the same for the content of terpenoids emitted from tobacco plants infested by $M$. persicae for 24 hours, when compared to healthy plants $\left(F_{2,6}=11.12, P=0.02\right)$ (Fig. 2). 


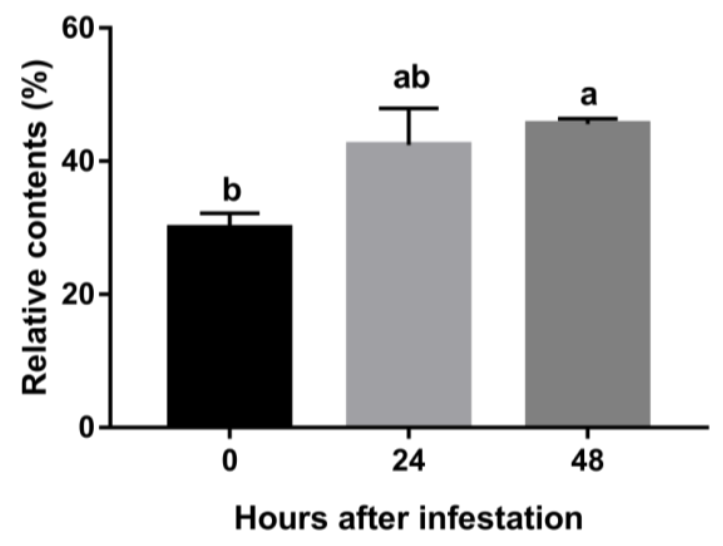

Figure 1. The total relative contents of released volatiles from tobacco after aphid-infestation for various durations. The different letters indicate the significant differences (Tukey's HSD test after ANOVA, $P<0.05$ )

(A) Aliphatic compound

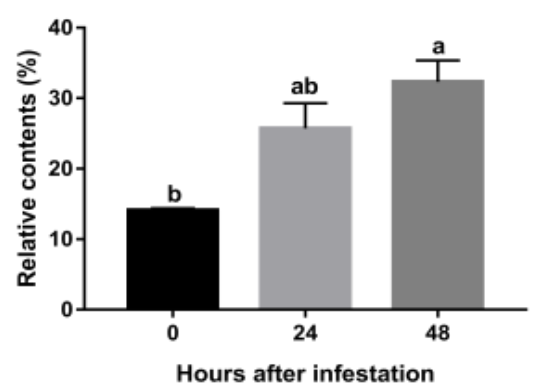

(B) Aromatic compounds

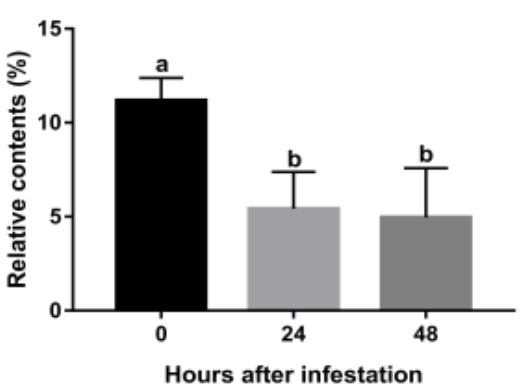

(C) Terpenoid

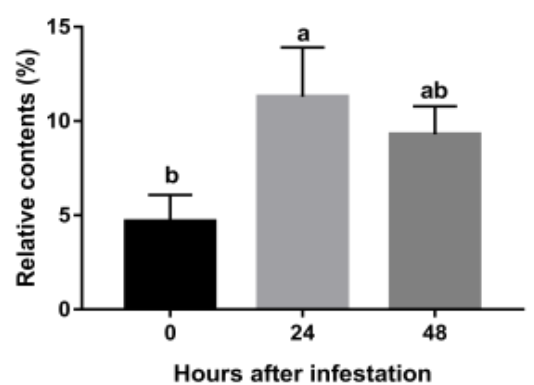

Figure 2. The relative contents of the different types of released volatiles from tobacco after aphid-infestation for various duration. The different letters indicate the significant differences (Tukey's HSD test after ANOVA, $P<0.05$ )

\section{Discussion}

In the present study, the investigators indicated that tobacco plants bearing the attack by $M$. persicae are activated to generate HIPVs that may have multiple roles as communication signals and defence agents against herbivores. The headspace profiles of plant volatiles between tobacco plants with and without $M$. persicae infestation are qualitatively and quantitatively different, and these were highly consistent with the features of HIPVs that have the high variability and diversity (Lou and Cheng, 2000). It 
has been widely accepted that the alterations of volatile release are one of the important and immediate responses of plants to insect herbivory (War et al., 2011; Aljbory and Chen, 2018; Erb and Reymond, 2019). The "chemical fingerprint" spectrum released by plants mediates the behaviors of insects, and the chemical volatile spectrum generally consists of numerous chemical components with different dosages (De Moraes et al., 1998; Runyon et al., 2020). For example, Takemoto and Takabayashi (2015) concluded that Aphidius ervi Haliday (Hymenoptera: Braconidae) are more attracted to the blend of plant volatiles induced by the host, when compared to each of the pure compounds. Thus, these distinct volatile compounds may play an important role in tobacco resistance against pests, and the natural enemies of attacking insects, or the surrounding of plants could perceive the qualitative and quantitative alterations of volatile compounds in aphid-infested plants in response to insect herbivore damage. Hence, the ecological function of the key components involved in these insect-plant interactions deserve further investigation.

Tobacco plants bear $M$. persicae feed with the newly produced 12 compounds, including tridecane, 1h-3a,7-methanoazulene, tetradecane, 1,8-nonadien-3-ol, pentadecane, hentriacontane, nonane, heneicosane, sulfurous acid, limonene, cedrene and dichloro acetaldehyde, and the later five volatile compounds were only isolated from tobacco plants at 48 hours after aphid infestation. Furthermore, the volatile compound found in both healthy tobacco plants and tobacco plants with $M$. persicae herbivory, such as the compound of undecane, was much more abundant in plant subjected to pests that have attacked for a long duration. A previous research demonstrated that Aphidius gifuensis Ashmead (Hymenoptera: Aphidiidae) is more attracted to the aphid/tobacco complex under Y-tube olfactometer determinations. Furthermore, the attractiveness was enhanced as the infestation levels increased, and this peaked at the aphid density of 400/plant (Yang et al., 2009). Additionally, Wu (2011) reported that $1 / 10$ of the crude extracts of aphid-infested tobacco plants could significantly repel $M$. persicae, but crude extracts diluted to $1 / 10,000$ could elicit a contrasting effect. Therefore, the investigators inferred that these specific chemical components may be more interesting due to the significant implications for plant protection in multitrophic contexts, which have been verified on some tobacco-pestnatural enemy systems, such as Tobacco, Lasioderma serricorne Fabricius (Coleoptera: Anobiidae) and Lariophagus distinguensis Foerster (Hymenoptera: Pteromalidae) (Lu et al., 2011).

$\mathrm{Wu}$ (2011) isolated undecane, dodecane, limonene and cedrene in headspace volatiles from both intact tobacco plants and plants with $M$. persicae infestation, while limonene and cedrene were detected only in tobacco plants with long durations of aphid-infestation, but not in the controls in the present study. Furthermore, undecane and dodecane were both released by infested or healthy tobacco plants. However, for the content of undecane, the delay emission in monoterpenes, such as limonene, have been demonstrated in other plants, such as lima bean leaves (Arimura et al., 2008). Increasing attention has been given to the newly emerged or significantly increased volatile components of plants induced by insect herbivory, which are considered as critical defence agents and communication signals, but are rarely noticed on vanished chemical components. The present study speculates that the neighbouring plants of attacked plants, and the natural enemies or companion of attacking pests may take action by perceiving the absence of a single volatile component or the blend of volatile components. Hence, it is interesting and warranted to research the ecological roles of 
vanished volatile components due to insect attacks within the framework of the agricultural system.

In addition, the headspace profiles of tobacco plants with or without pest infestation were partly inconsistent with the previous research conducted by Wu (2011). Many factors, such as the varieties, developmental stage, cultivation methods and cultivation conditions of tobacco plants, volatile collection, GC-MS apparatus and program setting, may attribute to this difference. The headspace profile of tobacco plants is known to differ among varieties. For instance, Zhang et al. (2019) reported that the components and contents of tobacco volatiles from seven varieties were significantly different. Furthermore, a total of 43 volatiles were detected in the cultivars of K326, which were greater than that in the present research. In the research conducted by $\mathrm{Wu}(2011)$, the tobacco plants of cultivars Cuibi 2 were used as the research subject, and the water-cultivating method was applied to breed tobacco plants, which were different with that in the present study. Furthermore, the methods of volatile collection and GC-MS analysis, including the GC-MS instrument and the procedure setting in their study, were different from those in the present study. For example, Tenax-Ta was utilized as volatile sorbent in that research, while Porapak Q was used in the present research. A previous study compared the adsorption efficiency of five volatile sorbents toward maize (Zea mays L.) leaves, and it was found that different types of sorbents have different affinities for different substances, Porapak Q had better affinity to terpenes, when compared to Tenax-Ta (Zhao et al., 2011). Furthermore, the infestation level of tobacco by aphids in the research conducted by $\mathrm{Wu}$ was 50 aphids per tobacco plant, which was significantly lesser than that in the present research (400 aphids per tobacco). Many research studies have indicated that the infestation levels of pests would significantly affect the emission of attacked plants (Lou and Cheng, 2000; Aljbory and Chen, 2018). This may result in qualitative and quantitative differences in the volatile release of attacked plants with different infestation levels.

In the present study, it was also found that the total content of volatiles components emitted from aphid-infested tobacco plants were higher than those from intact plants, which highly agrees with various studies conducted on other plants (Kessler and Baldwin, 2001; Heil, 2008; Arimura et al., 2008, 2009; Dicke et al., 2009). The types of these released components were more abundant than those from intact plants, particularly aliphatic compounds and terpenoids. Wu (2011) reported that the content of aliphatic compounds in tobacco plants, such as olefins and alcohols, significantly increased after being damaged by aphids. Thus, it was speculated that these two kinds of substances are the key components that act as insect repellents or natural enemies of attraction. Combined with the results of the previous study, the investigators deduced that these aliphatic and terpenoid compounds may play an important role in evoking the indirect defence mechanisms of tobacco plants. However, further verifications are needed.

\section{Conclusion}

The present study compared the headspace volatile profiles of tobacco plants, with or without aphid-infestation, at different time intervals by GC-MS. The total contents and types of tobacco volatiles increased after aphid infestation, and several specific components were newly emitted from aphid-infested tobacco plants. Understanding the ecological significance and roles of these specific HIPVs by combining biochemical and 
molecular mechanisms would open up a novel channel for further research projects on primary signalling cascades to the ecological consequences in multifarious ecosystems. The identification and characterization of volatile compounds that regulate the olfaction-directed behavior of pests and the natural enemies are needed to set-up tactics for developing cultivars with induced and constitutive resistance to pests. Furthermore, this would also assist in enhancing the effectiveness of natural enemies for pest control via the manipulation of such volatiles, in order to recruit these biological control agents for crop pests.

Acknowledgements. We would like to express our deepest gratitude to Nanping Tobacco Co. Ltd., Nanping, Fujian, China for providing the tobacco seeds. We would also like to thank Pro. Weiyi He (Institute of Applied Ecology, College of Plant Protection, Fujian Agriculture and Forestry University) for the technical guidance, and for providing the GC-MS. This research was funded by the Education and Scientific Research Project for Young and Middle-aged Teachers in Fujian Province (JAT190805, JAT190801), the Advanced Talents Introduction Project of Wuyi University (YJ201910), and Science \& Technology Innovation Platform Construction Project of Fujian Province (2018N2004).

Conflict of interests. The authors declare that they have no conflicts of interests. The funders had no role in the design of the study; in the collection, analyses, or interpretation of data; in the writing of the manuscript, or in the decision to publish the results.

\section{REFERENCES}

[1] Aljbory, Z., Chen, M. S. (2018): Indirect plant defense against insect herbivores: a review. - Insect Science 25(1): 2-23.

[2] Anfora, G., Tasin, M., Anfora, G., Tasin, M., Cristofaro, A. D., Ioriatti, C., Lucchi, A. (2009): Synthetic grape volatiles attract mated Lobesia botrana females in laboratory and field bioassays. - Journal of Chemical Ecology 35: 1054-1062.

[3] Arimura, G., Köpke, S., Kunert, M., Volpe, V., David, A., Brand, P., Dabrowska, P., Maffei, M. E., Boland, W. (2008): Effects of feeding Spodoptera littoralison Lima bean leaves: IV. Diurnal and nocturnal damage differentially initiate plant volatile emission. Plant Physiology 146: 965-973.

[4] Arimura, G., Matsui, K., Takabayashi, J. (2009): Chemical and molecular ecology of herbivore-induced plant volatiles: proximate factors and their ultimate functions. - Plant and Cell Physiology 50: 911-923.

[5] Bass, C., Puinean, A. M., Zimmer, C. T., Denholm, I., Field, L. M., Foster, S. P., Gutbrod, O., Nauen, R., Slater, R., Williamson, M. S. (2014): The evolution of insecticide resistance in the peach potato aphid, Myzus persicae. - Insect Biochemistry and Molecular Biology 51: 41-51.

[6] Blackman, R., Eastop, V. F. (2017): Taxonomic issues. - In: van Emden, H. F., Harrington, R. (eds.) Aphids as Crop Pests. Wallingford, England: CABI. pp. 1-36.

[7] Cai, P. M., Song, Y. Z., Huo, D., Lin, J., Zhang, H. M., Zhang, Z. H., Huang, F. M., Xiao, C. M., Ji, Q. E. (2020): Chemical cues mediating behavioral and electrophysiological responses of Fopius arisanus (Hymenoptera: Braconidae): the role of herbivore-induced plant volatiles. - Applied Ecology and Environmental Research 18(4): 5475-5489.

[8] De Moraes, C. M., Lewis, W. J., Paré, P. W., Alborn, H. T., Tumlinson, J. H. (1998): Herbivore-infested plants selectively attract parasitoids. - Nature 393(6685): 570-573.

[9] Dicke, M., van Loon, J. J. A., Soler, R. (2009): Chemical complexity of volatiles from plants induced by multiple attack. - Nature Chemical Biology 5: 317-324.

[10] Erb, M., Reymond, P. (2019): Molecular interactions between plants and insect herbivores. - Annual Review of Plant Biology 70: 527-557. 
[11] Faccoli, M., Anfora, G., Tasin, M. (2011): Stone pine volatiles and host selection by Tomicus destruens (Wollaston) (Coleoptera: Curculionidae, Scolytidae). - Silva Lusitana 19: 61-73.

[12] Frost, C. J., Mescher, M. C., Carlson, J. E., De Moraes, C. M. (2008): Plant defense priming against herbivores: Getting ready for a different battle. - Plant Physiology 146: 818-824.

[13] Gebreziher, H. G. (2018): The role of herbivore-induced plant volatiles (HIPVs) as indirect plant defense mechanism in a diverse plant and herbivore species: A review. International Journal of Agriculture and Food Science 2: 139-147.

[14] Heil, M. (2008): Indirect defence via tritrophic interactions. - New Phytologist 178: 4161.

[15] Hilker, M., Meiners, T. (2006): Early herbivore alert: insect eggs induce plant defense. Journal of Chemical Ecology 32: 1379-1397.

[16] Hilker, M., Fatouros, N. E. (2015): Plant responses to insect egg deposition. - Annual Review of Entomology 60(1): 493-515.

[17] Hong, F., Han, H. L., Pu, P., Dong, W., Wang, J., Liu, Y. H. (2019): Effects of five host plant species on the life history and population growth parameters of Myzus persicae (Hemiptera: Aphididae). - Journal of Insect Science 19(5): 15.

[18] Jackowski, J., Poploński, J., Twardowska, K., Magiera-Dulewicz, J., Hurej, M., Huszcza E. (2017): Deterrent activity of hops flavonoids and their derivatives against stored product pests. - Bulletin of Entomological Research 107: 592-597.

[19] Karban, R. (2011): The ecology and evolution of induced resistance against herbivores. Functional Ecology 25: 339-347.

[20] Kessler, A., Baldwin, I. T. (2001): Defensive function of herbivore-induced plant volatile emissions in nature. - Science 291: 2141-2144.

[21] Lou, Y. G., Cheng, J. A. (2000): Herbivore-induced plant volatiles: primary characteristics, ecological functions and its release mechanism. - Acta Ecological Sinica 20(6): 1097-1106.

[22] Lu, Y. J., Wang, Z. Y., Liu, J., Li, Y. Y. (2011): Effects of semiochemicals on the tritrophic interactions among the stored tobacco, Lasioderma serricorne (Coleoptera: Anobiidae) and Lariophagus distinguensis (Hymenoptera: Pteromalidae). - Proceedings of 2011 Inernational Symposium on Biomedicine and Engineering, Bali Island, Indonesia, pp. 432-435.

[23] Martin, B., Collar, L., Tjallingii, W. F., Fereres, A. (1997): Intracellular ingestion and salivation by aphids may cause the acquisition and inoculation of non-persistently transmitted plant viruses. - Journal of General Virology 78: 2701-2705.

[24] Philippi, J., Schliephake, E., Jurgens, H. U., Jansen, G., Ordon, F. (2015): Feeding behavior of aphids on narrow-leafed lupin (Lupinus angustifolius) genotypes varying in the content of quinolizidine alkaloids. - Entomologia Experimentalis et Applicata 156: 37-51.

[25] Phuong, T. T. H., Wróblewska-Kurdyk, A., Dancewicz, K., Gabryś, B. (2015): Selective acceptance of Brassicaceous plants by the peach potato aphid Myzus persicae: a case study of Aurinia saxatilis. - Acta Biologica 846: 51-62.

[26] Runyon, J. B., Gray, C. A., Jenkins, M. J. (2020): Volatiles of high-elevation five-needle pines: Chemical signatures through ratios and insight into insect and pathogen resistance. - Journal of Chemical Ecology 46(3): 264-274.

[27] Sharma, H. C., Sujana, G., Rao, D. M. (2009): Morphological and chemical components of resistance to pod borer, Helicoverpa armigera in wild relatives of pigeon pea. Arthropod-Plant Interaction 3: 151-61.

[28] Silva, A. X., Jander, G., Samaniego, H., Ramsey, J. S., Figueroa, C. C. (2012): Insecticide resistance mechanims in the green peach aphid Myzus persicae (Hemiptera: Aphididae) I: a transcriptomic survey. - PLoS One 7: e36366. 
[29] Takemoto, H., Takabayashi, J. (2015): Parasitic wasps Aphidius erviare more attracted to a blend of host-induced plant volatiles than to the independent compounds. - Journal of Chemical Ecology 41: 801-807.

[30] Turlings, T. C., Ton, J. (2006): Exploiting scents of distress: the prospect of manipulating herbivore-induced plant odours to enhance the control of agricultural pests. - Current Opinion in Plant Biology 9(4): 421-427.

[31] War, A. R., Sharma, H. C., Paulraj, M. G., War, M. Y., Ignacimuthu, S. (2011): Herbivore induced plant volatiles: their role in plant defense for pest management. - Plant Signaling and Behavior 6(12): 1973-1978.

[32] Wei, J. N., Li, T. F., Kuang, R. P., Wang, Y., Yin, T. S., Wu, X. F., Zou, L., Zhao, W. Y., Cao, J., Deng, J. H. (2003): Mass rearing of Aphidius gifuensis (Hymenoptera: Aphidiidae) for biological control of Myzus persicae (Homoptera: Aphidiidae). Biocontrol Science and Technology 13: 87-97.

[33] Wróblewska-Kurdyk, A., Nowak, L., Dancewicz, K., Szumny, A., Gabryśs, B. (2015): In search of biopesticides: the effect of caraway Carum carvi essential oil and its major constituents on peach potato aphid Myzus persicae probing behavior. - Acta Biologica 22: 51-62.

[34] Wu, P. (2011): Tobacco volatiles induced by Myzus persicae (Sulzer) infestation and their resistance to the aphid. - Master's thesis, Fujian Agriculture and Forestry University, Fuzhou, China.

[35] Yang, S., Xu, R., Yang, S. Y., Kuang, R. P. (2009): Olfactory responses of Aphidius gifuensis to odors of host plants and aphid-plant complexes. - Insect Science 16(6): 503510.

[36] Zhang, S. Q., He, J., He, W. J., Zhang, J. H., Zhou, F., Duan, D. W., Yang, T. Z. (2019): Comparative analysis of volatiles in different types of tobacco leaves. - Chinese Agricultural Science Bulletin 35(33): 52-57.

[37] Zhao, Z. J., Liu, T. X., Wang, X. P., Li, C. H. (2011): Adsorption efficiency of different adsorbents on volatiles of maize leaves. - Journal of Agricultural Science and Technology 13(2): 82-87. 